\title{
Impacts of a Web-based System on a Distributed Clinical Community of Practice
}

\author{
Marie Gustafsson Friberger \\ Department of Computer Science, School of Technology, Malmö University \\ Östra Varvsgatan 11A, SE-205 06 Malmö, Sweden \\ marie.friberger@mah.se
}

\begin{abstract}
SOMWeb is a Web-based system designed to support a clinical community of practice. Their collaboration centers on the discussion of oral medicine cases at monthly telephone conferences. SOMWeb facilitates the collaboration by, e.g., providing forms for entering case data and by providing easy access to current and previous cases. Through interviews, log analysis, and analysis of cases several impacts on the collaboration can be identified. These include the impact on membership, case submission, chairpersonship, and the community of practice as a whole. Further, it can be concluded that the rhythm provided by the telephone conferences ensures that the system has regular use.
\end{abstract}

\section{Introduction}

\subsection{The SOMNet Collaboration}

The starting point for this work is the problems associated with supporting the collaboration and improving the knowledge sharing of geographically and organizationally distributed clinicians in oral medicine. Oral medicine 1 is a subdiscipline of dentistry that often deals with disorders of low prevalence. Therefore, collaboration between clinics is needed to provide a means of consultation and learning for a broader audience. Further, collaboration is necessary to collect diverse and numerous cases for analysis.

For these reasons, the Swedish Oral Medicine Network (SOMNet) holds monthly telephone conference meetings to discuss difficult and interesting cases. SOMNet was initiated in the early nineties and, since then, they have used different variants of information technology to support their collaboration, such as e-mailing PowerPoint-files with case presentations.

One way of conceptualizing SOMNet is as a community of practice, a group of people who share "a concern, a set of problems, or a passion about a topic, and who deepen their knowledge and expertise in this area by interacting on an ongoing basis" [1. To be more precise, SOMNet is a distributed community

\footnotetext{
${ }^{1}$ Oral medicine concerns diseases related to the oral and paraoral structures. This includes the principles of medicine related to the mouth, as well as diseases specific to the orofacial tissues and oral manifestations of systemic diseases.
} 


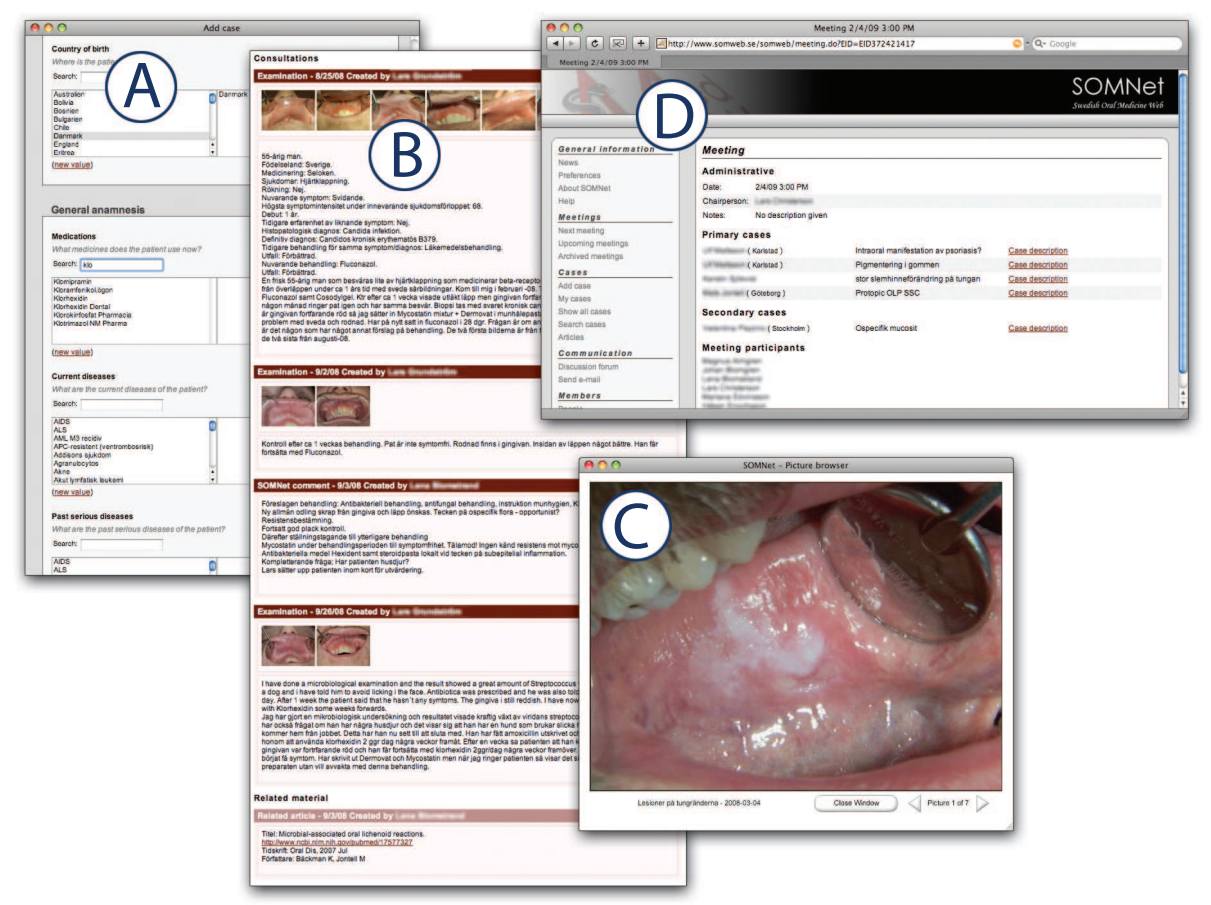

Fig. 1. The figure shows screenshots of some key parts of SOMWeb: part of an examination data entry form (A), case presentation with several consultations (B), the image browser for showing enlarged images (C), and a meeting page (D).

of practice, in that they are not co-located and seldom communicate through face-to-face communication.

\subsection{The SOMWeb System}

SOMNet's previous collaboration support had drawbacks such as difficulty in accessing old cases and a large variation in the information available for cases. A Web-based system, Swedish Oral Medicine Web (SOMWeb), was developed through a user-centered approach. SOMWeb and its development process are presented in Falkman et al. 2]. The system has been in use since May 2006. The SOMWeb system (see Fig. 11) includes functionality for case-entry, case browsing, and meeting support. Cases are entered using a community-defined case-entry form, which enables a more consistent data collection and that relevant information is available at the time of the meeting. SOMWeb also has functionality for assigning a chairperson to each meeting. Further, the chairperson can enter the group's recommendations to a discussed case, which is useful for case follow-up and for members who did not participate in the meeting. 
Table 1. Summary of figures for the data gathering methods.

\begin{tabular}{lll}
\hline Method & Data & Time period \\
\hline Interviews & 9 members & Nov 2007 - March 2008 \\
System data & n/a & May 2006 - Jan 2009 \\
System logs & n/a & Aug - Dec 2008 \\
Pre-SOMWeb cases 232 cases & Jan 2001-Apr 2006 \\
SOMWeb cases & 122 cases & May 2006 - Dec 2008 \\
\hline
\end{tabular}

\subsection{Article Overview}

This article analyzes the impacts of the SOMWeb system on the SOMNet collaboration. Following a description of the methods used (Sec. 2), the results of the evaluation (Sec. 3) and the identified impacts of the system on the collaboration (Sec. 4) are presented. The article ends with a discussion and conclusions (Sec. 5). Preliminary results regarding SOMWeb's impacts are presented in [2], but the analysis of logs and cases were not included in that article.

\section{Methods}

To evaluate SOMWeb, a variety of complementary methods were used: analysis of system data and usage logs, analysis of the cases submitted for discussion, as well as interviews. Table 1 summarizes figures related to the methods used.

To investigate system usage, user activity in the SOMWeb system was logged during the fall of 2008. Members of SOMNet were informed of the logging on the page users reach upon logging in. The logs were internally created by the system, and each log line consists of the date and time, user id, as well as the page requested.

Cases from before and after the introduction of SOMWeb were analyzed to examine the distribution of submitting clinics. The included cases are those presented from approximately 2000 until the introduction of SOMWeb in May 2006 (approximately 230 cases), as well as those contained within SOMWeb (approximately 120 cases at the time of analysis). To be able to compare the distribution of submitting clinics, data on the name of the submitter and their clinic were noted for all pre-SOMWeb cases. For the SOMWeb cases, this information was automatically extracted.

A semi-structured interview format was chosen and the interview guide included parts about submitting cases, meeting participation and preparation, and use of the SOMWeb system outside of meetings. The interviewees were selected in order to get respondents from different clinics and with different lengths of membership. Nine members were interviewed. Each interview lasted between 35 and 85 minutes. The interviews were recorded and transcribed. Patterns in the interview data were identified and coded. The interview questions were used as initial coding themes. 
Since data gathering and analysis were performed by one of the system developers, there is the possibility for bias in more readily seeing results that confirm the intent and proper functioning of the system. The behavior interviewees could have been affected by them knowing that I had taken part in the development.

\section{Results}

\subsection{Membership and Case Submission}

In January 2009, when the evaluation period was over, SOMWeb had 120 registered users located at 74 clinics. It had been used at 23 meetings and the case repository contained 121 cases 2 When SOMWeb was introduced in May 2006, it had about 40 members, all hospital dentists. At the time of the evaluation, the membership included private practice and public clinic dentists as well. To give further indication of the number of members during the evaluation period, there were 86 members in February 2008. About $25 \%$ of the members had submitted at least one case. Five people had submitted about $50 \%$ of the 121 cases in the repository.

\subsection{System Use and Benefits}

Interviewees indicate using SOMWeb mainly during meetings and in preparation for these. A few days before the meeting or on the day of the meeting they examine cases in the system to form their own opinion about them. Interviewees also reported that they had logged in to browse previous cases. One of these stated that he had never done so for the previous PowerPoint-based approach.

The analysis of logs also indicates that the system is mainly used in conjunction with the meetings, as seen in Fig. 2, which displays the number of unique logins (that is, a user logging in several times is only counted once) per day. There are four peaks, corresponding to the dates of meetings. The number of logins per meeting day does not reflect the number of active members, since one clinic often has several participants where only one is logged in.

During the first year of use of SOMWeb, the same person chaired all meetings and due to a lack of time only rarely entered meeting notes. Since the decision in June 2007 that the chair should circulate, interviewees found meeting preparations more thorough. For the fall of 2007 and spring of 2008 notes were added for most cases at five out of eight meetings.

All those interviewed stated that the SOMWeb system has improved the SOMNet collaboration. Motivations include making case entry easier and less time-consuming, prompting the supply of more uniform case data, enabling a collected view of a case over time, and providing more structure to SOMNet's activities in general.

\footnotetext{
${ }^{2}$ To give an indication of its use since, in June 2010, SOMWeb had 248 registered users at 169 clinics, had been used at 37 meetings and contains 183 cases.
} 


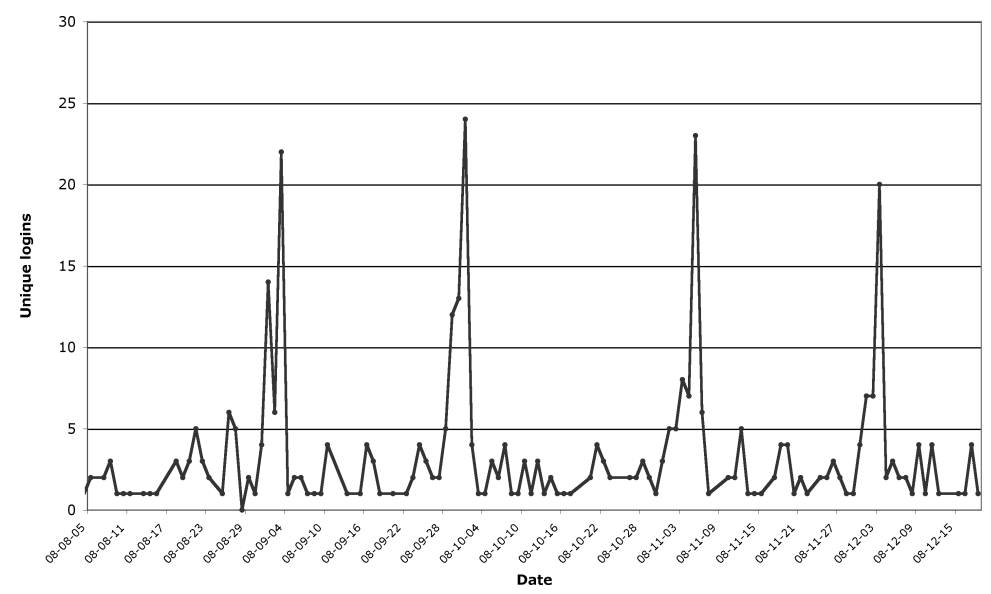

Fig. 2. Unique logins to SOMWeb during the fall of 2008. The peaks correspond to SOMNet meetings.

\subsection{Distribution of Submitting Clinics}

The pre-SOMWeb cases were submitted by twelve clinics, all located at hospitals. For the SOMWeb cases, sixteen clinics have submitted cases, and while almost all of the submitters are hospital clinics, two are private practices and two are public dental care clinics. The same three clinics have the most number of cases both for the pre-SOMWeb and SOMWeb cases, and these clinics have been part of the SOMNet collaboration since its inception. Before SOMWeb, the cases from these clinics represented $63 \%$ of all cases. Since SOMWeb's introduction, cases from these clinics represent $51 \%$ of all cases. Fig. 3 shows the number of cases per clinic, before and after the introduction of SOMWeb.

\section{Impacts}

\subsection{Impact on SOMNet as a Community of Practice}

A community of practice has a shared repertoire, the common resources that it produces [4]. For SOMNet, the primary artifacts are the cases, first as e-mailed files and then as structured case presentations. With SOMWeb, this shared repertoire is more easily and continually available. When files were e-mailed, newly joined members did not obtain access to old cases. Further, other kinds of information entities can now be included, such as meeting decisions and articles.

Communities of practice have varying levels of formality [1. SOMNet has previously been recognized as a collaboration between colleagues at different workplaces. With the introduction of SOMWeb, the membership has become more clear, and to some extent the community has become more formal. 


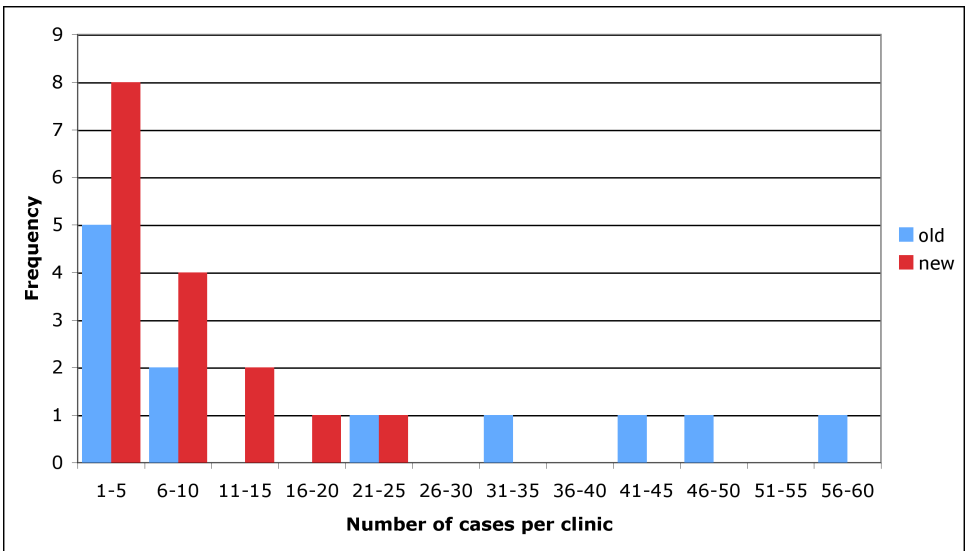

Fig. 3. Frequency distribution of number of cases per clinic, before and after the introduction of SOMWeb. The x-axis shows intervals for the number of cases per clinic, such as one to five cases, and the y-axis shows the number of clinics within that interval.

\subsection{Impact on Membership and Participation}

When SOMNet's activities started more than ten years ago, four clinicians participated in the case discussions. At the beginning of this century, PowerPointpresentations were distributed to less than ten participants, all specialists in oral medicine. SOMNet comprised a small group of active clinicians who all participated in the discussions. For the first 2.5 years of system use, SOMWeb had a three-fold increase in registered members. New members may have become aware of SOMWeb through presentations and outreach activities of core members, through word of mouth, and articles in professional media. One can speculate that the membership increase would have been more difficult without a Web collaboration system to which prospective members could be referred.

The community of practice literature discusses the existence of different levels of participation [1, where participants can be divided into the groups of core, active, and peripheral. Many participants often belong to the last group, which mostly observes interactions between core and active members. Compared to before SOMWeb, more general practitioner dentists are now members, which is connected to the increase of the number of peripheral participants with the introduction of SOMWeb, and this has enabled the spread of oral medicine expertise beyond the core and active groups.

\subsection{Impact on Case Submission}

Several clinics still dominate the submission of cases, but there is an indication that this prevalence is lower with SOMWeb than previously. SOMNet aims to aid more clinics and to spread oral medicine awareness. Increasing the participation of clinics where such activities are not a core part of work signifies a step 
towards achieving this goal. That seven clinics that had not submitted cases before SOMWeb have now submitted cases may indicate that SOMWeb enables the participation of a wider range of clinics.

\subsection{Impact on Chairpersonship and Recording Meeting Decisions}

The rotation of chairpersonship has several benefits. It reduces pressure on the original chairperson, which means that notes on meeting recommendations can be more consistently entered to SOMWeb cases. It also suggests that more members feel involved in the work of SOMNet, and that knowledge of how this work is carried out is spread to more people. Since this amounts to more external evidence, such as article references, being added, those clinicians less experienced in searching and using literature get more such exposure. However, the recording of meeting recommendations is not always performed. Reasons for this include a lack of time and not knowing that it should be done. To alleviate the latter problem, this information is more clearly available.

\section{Discussion and Conclusions}

The SOMWeb system's impacts on SOMNet's collaboration include enabling the participation of a wider range of clinics. Factors influencing this can be the more accessible submission process as well as the increased tangibility and formality that a Web system gives over e-mailing. This also makes outreach to potential new participants easier, and indeed the membership of SOMNet has grown since SOMWeb's introduction. This expansion is probably also part of the natural growth of a healthy community of practice. However, the technical support must be in phase with the community growth, and the initial procedure probably could sustain fewer members than SOMWeb. Further, as the membership grows, it is less likely that members personally know each other. Thus, the increased visibility of SOMNet's members in SOMWeb, via lists of members and what clinic they work at, becomes a necessity.

The analysis of logs shows that activity peaks on meeting days. This is to be expected, as the system was explicitly designed to support these meetings. That SOMNet has this regular activity means the system is continuously being used, although its use is not evenly distributed throughout the month. However, one can wonder whether or not this is desirable. On the one hand, the meetings provide a rhythm for the collaboration, a desirable property of a community of practice [1. The usage patterns of SOMWeb point to a successful alignment with this rhythm. On the other hand, increased use of SOMWeb between meetings, for example by clinicians seeking information related to current cases, would heighten the everyday clinical benefit of the system. Further investigation is needed to examine the activities in SOMWeb between meetings, and to ascertain when and how SOMWeb can be used in daily work.

SOMNet's forms of collaboration played a major role in the design of SOMWeb, while SOMWeb in turn has affected SOMNet's collaboration. This 
is in line with the conclusion of Koch and Fusco [5] that the choice of tools to support a community will affect its social components. In the case of SOMNet, the participants' already established collaboration probably affected the actual use of SOMWeb positively. However, given that SOMNet's collaboration bears similarities with other forms of medical collaborations, such as the multidisciplinary medical team meetings described by Kane and Luz [6], it is possible that the system can be used to bootstrap a new collaboration.

Another factor that has affected SOMWeb's continued use is the presence of a champion who has been active during its development, introduction, and continued use. This is in line with Lock Lee and Neff [7, who conclude that the introduction of new technologies needs a champion within the community. Thus, if the SOMWeb system is used for other groups of collaborating clinicians, the identification of a champion is likely to be necessary for success.

A community of practice, with its shared repertoire, often has specific artifacts around which its problem-solving and learning revolves. For SOMNet, the case is a central artifact, which therefore has a focal role in SOMWeb. When constructing a system to support distributed communities of practice, the identification of their core artifacts cannot be overlooked, and the reinforcement of this in any system of support is essential.

\section{References}

1. Wenger, E., McDermott, R., Snyder, W.: Cultivating Communities of Practice. Harvard Business School Press, Boston, MA (2002)

2. Falkman, G., Gustafsson, M., Jontell, M., Torgersson, O.: SOMWeb: A Semantic Web-based system for supporting collaboration of distributed medical communities of practice. J Med Internet Res 10(3) (Aug 2008) e25

3. Patton, M.Q.: Qualitative Evaluation and Research Methods. 2nd edition edn. SAGE Publications (1990)

4. Wenger, E.: Communities of Practice: Learning, Meaning, and Identity. Cambridge University Press, Cambridge, U.K. and New York, N.Y. (1998)

5. Koch, M., Fusco, J.: Designing for growth: Enabling communities of practice to develop and extend their work online. In Kimble, C., Hildreth, P.M., eds.: Communities of Practice: Creating Learning Environments for Educators. Information Age Publishing Inc., Greenwich, CT (2007)

6. Kane, B., Luz, S.: Multidisciplinary medical team meetings: An analysis of collaborative working with special attention to timing and teleconferencing. Computer supported cooperative work 15(5) (2006) 501-535

7. Lock Lee, L., Neff, M.: How information technologies can help build and sustain an organization's CoP: Spanning the socio-technical divide. In Hildreth, P.M., Kimble, C., eds.: Knowledge Networks: Innovation Through Communities of Practice. IGI Publishing (2004) 